\title{
Fibrose rétropéritonéale : revue de littérature
}

\author{
Nabil Jakhlal, MD; Anouar Elghazoui, MD; Youness Jabbour, MD; Tarik Karmouni, MD; Khalid Elkhader, MD; \\ Abdellatif Koutani, MD; Ahmed Ibenattya, MD
}

Service d'Urologie B, hôpital Ibn Sina de Rabat, Maroc

Cite as: Can Urol Assoc J 2017;11 (1-2):E26-31. http://dx.doi.org/10.5489/cuaj.4122

Published online January 12, 2017

\section{Résumé}

La fibrose rétropéritonéale (FRP) est une maladie rare, caractérisée par la présence d'un tissu fibro-inflammatoire aberrant qui se forme souvent autour de la portion sous-rénale de l'aorte abdominale et des artères iliaques. Une obstruction urétérale est souvent observée. Récemment, la FPR idiopathique est rapportée parmi les manifestations de la maladie à IgG4. L'imagerie joue un rôle important dans le diagnostic de la FRP. Les glucocorticoïdes sont le traitement de référence, utilisés en monothérapie ou en association avec d'autres agents médicamenteux. En cas d'échec des mesures conservatrices, la chirurgie peut être proposée. Récemment, des techniques minimalement invasives ont été utilisées.

\section{Introduction}

La fibrose rétropéritonéale (FRP) est une maladie rare, caractérisée par la présence d'un tissu fibro-inflammatoire aberrant qui se forme souvent autour de la portion sous-rénale de l'aorte abdominale et des artères iliaques. Elle engaine fréquemment les structures avoisinantes : les uretères et la veine cave inférieure. ${ }^{1}$ Cette maladie a été décrite pour la première fois par l'urologue français Albarran en 1905, mais la description de deux cas par Ormond en 1948 a permis de la considérer comme une entité clinique. ${ }^{2,3}$ Elle est idiopathique dans deux tiers des cas, le tiers restant peut être secondaire à des infections, une chirurgie abdominale, la prise de médicaments ou des tumeurs malignes. Récemment, la FPR idiopathique est rapportée parmi les manifestations de la maladie à IgG4.

L'objectif de cette étude est de faire le point par rapport à de récents articles publiés concernant la physiopathologie, le diagnostic et le traitement de la FPR.

\section{Méthodologie}

À partir d'une base de données électronique (par exemple, PubMed), une recherche dans la littérature a été effectuée en utilisant des mots clés pertinents, comme fibrose rétropéritonéale, maladie d'Ormond, périaortite chronique ou fibrose rétropéritonéale liée à la maladie à lgG4. La majorité des articles utilisés dans notre travail comme référence ont été publiés au cours de la dernière décennie.

\section{Étiologie}

La distinction entre les formes idiopathiques et secondaires de la FPR est faible, car le lien de causalité direct entre la cause secondaire et la maladie est peu claire. Certains auteurs considèrent ces conditions (notamment la chirurgie, la radiothérapie et les médicaments) comme des facteurs prédisposants. ${ }^{4}$

La FPR secondaire peut être le résultat $d^{\prime}$ un anévrisme de l'aorte, d'une radiothérapie antérieure, d'une chirurgie abdominale, de traumatismes, de tumeurs rétropéritonéales primaires ou métastatiques, d'infections (tuberculose, histoplasmose, actinomycose) et de la prise de médicaments (ergotamine, méthyldopa, bêta-bloquants, méthysergide, bromocriptine, hydralazine) (tableau 1). ${ }^{5}$ Les tumeurs rétropéritonéales primaires, tels que le lymphome ou les sarcomes et les tumeurs métastatiques, tels que le cancer du sein et du côlon, peuvent aussi produire une réaction fibro-inflammatoire et ainsi imiter la FRP. ${ }^{6}$ La FPR maligne est d'une importance particulière en raison de son mauvais pronostic. Sa fréquence est estimée à $8 \%$ des cas. ${ }^{7}$

\section{Étiopathogenèse}

La pathogenèse de la FPR est encore obscure. En 1980, Parums et Mitchinson considéraient la FRP comme une réaction inflammatoire locale exagérée à l'athérosclérose aortique. Cette réaction est déclenchée contre des lipides contenus dans la plaque d'athérosclérose comme des LDL oxydés et des céroïdes. ${ }^{8}$ 


\begin{tabular}{|c|c|}
\hline Médicaments & $\begin{array}{l}\text { Méthysergide } \\
\text { Pergolide } \\
\text { Bromocriptine } \\
\text { Ergotamine } \\
\text { Méthyldopa } \\
\text { Hydralazine } \\
\text { Analgésiques } \\
\text { Bêta-bloquants }\end{array}$ \\
\hline Tumeurs malignes & $\begin{array}{c}\text { Tumeurs carcinoïdes } \\
\text { Lymphomes Hodgkiniens et non } \\
\text { Hodgkiniens } \\
\text { Sarcomes } \\
\text { Cancers : côlon, prostate, poumon, } \\
\text { estomac }\end{array}$ \\
\hline Infections & $\begin{array}{c}\text { Tuberculose } \\
\text { Histoplasmose } \\
\text { Actinomycose }\end{array}$ \\
\hline Radiothérapie & $\begin{array}{l}\text { Séminome testiculaire } \\
\text { Carcinome du côlon } \\
\text { Carcinome pancréatique }\end{array}$ \\
\hline Chirurgie & $\begin{array}{c}\text { Lymphadénectomie } \\
\text { Colectomie } \\
\text { Hystérectomie } \\
\text { Anévrysectomie aortique }\end{array}$ \\
\hline Autres & $\begin{array}{c}\text { Histiocytose } \\
\text { Maladie de l'Erdheim-Chester } \\
\text { Amylose } \\
\text { Traumatismes } \\
\text { Lavement baryté }\end{array}$ \\
\hline
\end{tabular}

Cependant, les patients atteints de FRP ont souvent des symptômes généraux, des taux élevés des marqueurs de I'inflammation, des auto-anticorps positifs, des maladies autoimmunes connexes, une atteinte d'autres organes et une bonne réponse aux immunosupresseurs. Ces résultats portent à croire que la FRP est une manifestation d'une maladie systémique plutôt qu'une réaction locale exagérée à l'athérosclérose. ${ }^{6,9}$

Certaines études supposaient que l'origine de la FRP était une aortite primaire suscitant par la suite une réponse fibroinflammatoire péri-aortique. En accord avec cette hypothèse, I'inflammation vasculaire peut toucher d'autres segments vasculaires (l'aorte thoracique et ses branches, les artères gastro-intestinales) et prédominer au niveau de l'adventice ; une vascularite des vasa varum peut aussi être observée. ${ }^{10,11}$

Récemment, un certain nombre d'auteurs ont laissé entendre que la FPR idiopathique est en partie due à une affection inflammatoire systémique connue sous le nom de maladie à $\operatorname{lgG} 4 .{ }^{12}$ Celle-ci a été identifiée dans $47-58 \%$ des patients ayant déjà reçu un diagnostic de FPR idiopathique. ${ }^{13,14} \mathrm{De}$ même, environ $50 \%$ des anévrismes inflammatoires de l'aorte abdominale sont liés aux $\operatorname{IgG} 4 .{ }^{15}$ Cependant, nous ne disposons que de peu d'informations sur la pathogenèse de cette maladie et la relation entre les formes de la FRP liée et non liée aux IgG4.

\section{La FRPI ef la maladie à IgG4}

Le syndrome d'hyper-lgG4, ou maladie systémique associée aux IgG4, est une entité de description récente caractérisée par la présence d'une ou plusieurs atteintes fibro-inflammatoires d'organes, associée le plus souvent à une élévation des IgG4 sériques. Les lésions tissulaires sont caractérisées par une fibrose et un infiltrat inflammatoire lymphocytaire et plasmocytaire, avec prédominance de plasmocytes lgG4+.

Les atteintes organiques rapportées sont nombreuses et peuvent être associées chez un même patient. Parmi ces atteintes, on rapporte des tableaux de pancréatite sclérosante, de sialadénite, de dacryoadénite, de polyadénopathies, d'aortite, de cholangite sclérosante, de néphrite interstitielle, de fibrose rétropéritonéale ou encore de pseudotumeurs inflammatoires. L'évolution est habituellement marquée par une grande corticosensibilité. Deux groupes japonais ont développé en 2011 des critères diagnostiques plus complets pour les maladies à lgG4 (tableau 2). ${ }^{12}$

La maladie à lgG4 est responsable seulement d'une partie des cas de FRPI (50\%). ${ }^{13,14}$

La possibilité d'un terrain prédisposé, voire de facteurs génétiques, a été envisagée. Des études génétiques ont montré une forte association entre FRPI et HLA-DRB $1 * 03$, allèle lié à de nombreuses MAI telles que le diabète de type 1 , la myasthénie grave et la thyrö̈dite autoimmune. ${ }^{16}$ Le polymorphisme du gène de CCR5 expose à un risque accru de présenter une $\mathrm{FRPI}$, surtout dans sa forme anévrysmale. ${ }^{17}$

\section{Diagnostic}

\section{Clinique}

Les signes cliniques sont non spécifiques, très variables et souvent liés à l'effet mécanique de la FRP sur les structures avoisinantes : douleurs abdominales, lombaires (80\%), coliques néphrétiques, OMI, TVP, douleurs testiculaires, hydrocèle, claudication intermittente des MI. Des

\section{Tableau 2. Les critères diagnostiques pour les maladies à IgG4 ${ }^{12}$}

1. L'examen clinique révèle une tuméfaction diffuse/localisée ou une masse dans un ou de nombreux organes.

2. Le laboratoire montre un taux sérique élevé d'lgG4 (135 mg/dL).

3. Histopathologie :

a. infiltration marquante par lymphocytes et plasmocytes, fibrose

b. infiltration par plasmocytes IgG4 positifs avec quotient cellules IgG4/lgG positif $>40 \%$ et $>10$ plasmocytes $/ \mathrm{hpf}$

Diagnostic définitif : tous les 3 critères

Diagnostic propable : critères 1 et 3

Diagnostic possible : critères 1 et 2 plus exclusion d'un autre diagnostic 
signes généraux (fatigue, anorexie, amaigrissement...) sont fréquents, reflétant la nature inflammatoire de la maladie. ${ }^{1}$

$L$ présentation clinique initiale ne permet donc pas en règle générale de différencier la FRP idiopathique des formes secondaires. Il est donc important de rechercher systématiquement la notion d'exposition professionnelle à l'amiante, de tabagisme, la prise de médicaments potentiellement inducteurs de fibrose, la notion d'une radiothérapie ou d'une chirurgie antérieure ou encore les manifestations cliniques de la maladie à IgG4 touchant d'autres parties du corps..$^{12,15,18}$

\section{Biologie}

L'exploration biologique de la FRP trouve, classiquement, une élévation des marqueurs de I'inflammation associée à une anémie inflammatoire et une altération de la fonction rénale. La valeur de ces données doit être consignée, et servira de référence pour la surveillance de l'évolution de la maladie sous traitement. ${ }^{19}$ Dans une large cohorte de 58 patients atteints de FRP, I'augmentation de la VS et la CRP a été trouvé dans $66,7 \%$ et $64,9 \%$ des cas, respectivement. ${ }^{20}$ Les taux de créatinine varient en fonction de la gravité de l'obstruction urétérale. L'insuffisance rénale est objectivée dans 80 à $100 \%$ des cas. $^{1}$

Les anticorps anti-nucléaires (ANA) sont positifs chez 5 à $60 \%$ des patients, ${ }^{9,21}$ mais d'autres auto-anticorps peuvent également être positifs, y compris le facteur rhumatoïde, les anticorps cytoplasmiques anti-neutrophiles, anti-cellules musculaires lisses et anti-anticorps anti-thyrö̈diens. ${ }^{1,9,22}$ Cependant, les anticorps positifs ne présagent pas nécessairement la présence ou l'apparition des manifestations cliniques des maladies associées à ces auto-anticorps. ${ }^{6}$

Les IgG4 sériques peuvent être élevées chez les patients avec FRP liée à la maladie à $\operatorname{lgG} 4 .{ }^{13}$ Cette élévation des IgG4 sériques ne semble pas spécifique. En effet, celle-ci a été rapportée chez $5 \%$ des patients atteints du syndrome de Churg-Strauss, de pneumonie à éosinophiles, de la maladie de Castleman systémique. ${ }^{23,24}$

\section{Imagerie}

L'imagerie joue un rôle important dans le diagnostic de la FRP, dans la distinction des formes bénignes de bon pronostic des formes malignes de mauvais pronostic, et enfin dans le suivi de l'évolution sous traitement.

L'échographie est l'examen radiologique souvent réalisé en première intention, a une faible sensibilité pour la détection de la FRP, mais permet d'objectiver I'uropathie obstructive. $^{25,26}$

Pour le diagnostic et la distinction entre les formes bénignes et malignes, la TDM et I'IRM sont les examens de choix. Elles permettent de mettre en évidence la plaque de fibrose, de préciser sa morphologie, son emplacement et sa propagation aux structures avoisinantes. ${ }^{26,27}$ Elles peuvent également révéler d'autres atteintes de la maladie à IgG4 : pancréatite autoimmune, péricardite chronique avec fibrose médiastinale, etc. L'aspect typique est une masse bien limitée mais irrégulière du tissu péri-aortique qui s'étend du niveau des AR aux vaisseaux iliaques, et progresse dans le rétropéritoine en enveloppant les uretères et la $\mathrm{VCl} .^{22,28,29} \mathrm{La}$ masse occupe les faces antérieure et latérales de l'aorte en épargnant sa face postérieure. ${ }^{22,28}$ Cependant I'apparence et l'étendue varient considérablement. Le tissu fibro-inflammatoire peut s'étendre en bas vers le pelvis, ${ }^{22}$ en haut audessus du hile rénal, ou dans de rares cas, il peut envahir le duodénum, ${ }^{30}$ le hile rénal ou le rein..$^{31,32}$

Plusieurs élements peuvent aider à faire la distinction :

- Le déplacement antérieur de l'aorte et de la veine cave inférieure est souvent vu dans la FRP maligne. Une hypertrophie des ganglions lymphatiques postérieurs aux gros vaisseaux pourrait expliquer cette donnée. Celle-ci manque de sensibilité et spécificité et donc sa généralisation n'est pas toujours correcte.

- Un emplacement rétropéritoneal céphalique. Les lymphomes sont souvent observés dans un endroit plus céphalique dans le rétropéritoine, alors que la FPR bénigne est principalement située au-dessous du hile rénal. ${ }^{27}$ Néanmoins, la propagation crâniale de la FPR bénigne au-dessus du hile rénal n'est pas rare. Par conséquent, l'étendue de la masse est d'une utilité clinique limitée. ${ }^{26}$

- Aspect nodulaire avec effet de masse sur les organes avoisinants

- La FRP bénigne dans ses stades précoces et la FPR maligne peuvent se rehausser après injection du produit de contraste. ${ }^{29}$ Par conséquent, le rehaussement n'est pas utile dans le diagnostic différentiel entre la maladie bénigne et maligne.

L'intérêt à faire un suivi par TDM ou IRM réside dans l'évaluation de la taille de la masse.

La TEP est une modalité d'imagerie fonctionnelle bien établie en oncologie, et de plus en plus utilisée dans l'évaluation des maladies inflammatoires, y compris la FRP. ${ }^{33-35}$

Elle a l'avantage d'examiner le corps entier objectivant ainsi d'autres sites inflammatoires (fibrosclérose multifocale, FRP secondaires aux processus néoplasiques ou infectieux). ${ }^{36,37}$

Par rapport aux marqueurs biologiques de l'inflammation et à l'imagerie morphologique, le 18F-FDG apparaît comme une modalité plus sensible pour l'évaluation de l'activité métabolique des masses résiduelles et la démonstration de la rechute de la maladie. ${ }^{37,38}$ 


\section{Biopsie}

La biopsie n'est pas toujours réalisée, surtout si les caractéristiques radiologiques sont en faveur d'une FPR. Toutefois, si les dilemmes diagnostiques existent et une tumeur maligne sous-jacente est soupçonnée ou s'il n'y a pas de réponse au traitement initial, la biopsie doit être effectuée pour que le diagnostic soit confirmé. La biopsie peut être effectuée chirurgicalement ou sous contrôle par imagerie. ${ }^{1,39}$

\section{Traitement}

Les objectifs thérapeutiques sont multiples :

- Traiter les complications obstructives comme une hydronéphrose unilatérale et/ou bilatérale.

- Diminuer le syndrome inflammatoire.

- Limiter la progression de la fibrose.

- Prévenir les récidives.

\section{Traitement médical}

Le traitement de la FRP idiopathique reste encore empirique et calqué sur la prise en charge des maladies autoimmunes ou inflammatoires.

Des cas anecdotiques de résolution spontanée ont été rapportés ${ }^{40}$ et quelques patients asymptomatiques sans répercussion sur les structures adjacentes pourraient relever uniquement d'un suivi.

Pour les patients symptomatiques, la corticothérapie reste le traitement de première intention, mais il n'existe aucun consensus concernant la dose $(0,5-1 \mathrm{mg} / \mathrm{kg} / \mathrm{jour})$ et la durée. Les protocoles les plus rapportés dans la littérature sont :

- $60 \mathrm{mg} /$ jour pendant deux mois (1j/2), puis diminution progressive à $5 \mathrm{mg}$ en deux mois, puis $5 \mathrm{mg}$ /jour pendant deux ans ${ }^{41}$

- $\quad 40-60 \mathrm{mg}$ /jour pendant six semaines puis diminution progressive sur 2-3 mois pour atteindre 5-10 mg. Durée : 6-12 mois $^{39}$

- $1 \mathrm{mg} /$ jour (max $80 \mathrm{mg}$ ) pendant quatre semaines, puis diminution progressive pour atteindre $10 \mathrm{mg} /$ jour en 2-3mois. Durée : 12-18 mois $^{1}$

Les auteurs ont conclu à une efficacité rapide des corticoïdes sur les symptômes cliniques, les paramètres biologiques et plus rarement les images radiologiques, ce qui en fait un bon traitement d'induction. Cependant, leur utilisation au long cours entraînait plus d'effets indésirables que le tamoxifène.

Le tamoxifène, par ses propriétés anti-fibrosantes, peut être utilisé en relais des corticoïdes pour une épargne cortisonique. Un seul essai prospectif comparant l'efficacité de la prednisone contre le tamoxifène dans la prévention des récidives a été mené. Cette étude a montré que le tamox- ifène est moins efficace que la prednisone en présence de récidives. ${ }^{42}$

D'autres stratégies d'épargne cortisonique ont été essayées avec le mofétilmycophénolate, le cyclophosphamide et I'azathioprine. ${ }^{43-45}$ Les stratégies associant la prednisone et le mofétilmycophénolate ou l'azathioprine ont donné de bons résultats. Par contre, les effets secondaires dans la plus large étude associant cyclophosphamide et corticoïdes sont probablement inacceptables.

Bien que différents agents immunosuppresseurs aient été utilisés avec succès pour traiter la FRP, ${ }^{45,46}$ aucun d'entre eux n'a été systématiquement étudié chez les patients en rechute. Récemment, une étude a montré que l'association du méthotrexate et de la prednisone est une option viable pour traiter la FRP récurrente. Ce régime a amené une rémission chez une proportion considérable de patients porteurs de marqueurs de l'inflammation normalisés, une fonction rénale excellente et des effets toxiques liés au traitement limités. Après l'arrêt du traitement, les patients peuvent être à risque élevé de rechute et devraient être étroitement suivis. Plus récemment, des biothérapies ont été également utilisées dans le traitement de la FRP réfractaire. Ces agents peuvent avoir un rôle, mais des études plus importantes sont nécessaires. ${ }^{47}$

\section{Traitement chirurgical}

En cas de dilatation de la voie excrétrice urinaire, la désobtruction par sonde double J ou la néphrostomie percutanée s'imposent durant la phase aiguë en association avec le traitement médicamenteux. Les endoprothèses urétérales à demeure sont la forme la plus commune de drainage ; elles sont habituellement retirées après 3 mois de traitement.

Une fois la maladie stabilisée par le traitement médicamenteux et si l'obstruction urinaire patente persiste, la chirurgie peut être proposée. Elle permet l'urétérolyse avec biopsies profondes de la plaque, puis protection des uretères par repositionnement latéral avec intrapéritonéalisation et/ou utilisation d'un lambeau épiploïque ou péritonéal, obligatoires afin de restaurer la fonction rénale. ${ }^{1,46,48}$

L'approche chirurgicale peut être réalisée soit à ciel ouvert ou par voie laparoscopique avec ou sans assistance robot. Les techniques minimalement invasives ont un taux de complications similaires à la méthode classique et sont aussi efficaces. De plus, elles peuvent fournir une convalescence plus courte et un recours moindre à l'analgésie postopératoire et à la transfusion sanguine. ${ }^{49,50}$

Cependant, les procédures chirurgicales peuvent être associés à des risques importants de complications, y compris les plaies urétérales, une dévascularisation urétérale et des rétrécissements, la formation de fistules urinaires, une fibrose récurrente et des complications thromboembolique. ${ }^{26}$ 


\section{Conclusion}

La FRP est une maladie rare, le plus souvent idiopathique. La démarche diagnostique n'est pas codifiée; cependant la réalisation systématique d'une biopsie ne semble pas justifiée en I'absence d'éléments cliniques et/ou paracliniques d'orientation. Malgré l'absence de consensus, les corticoïdes associés au drainage urinaire en cas de dilatation de la voie excrétrice représenteraient le traitement de première intention. L'utilisation de certains immunosuppresseurs comme moyen d'épargne cortisonique a donné de bons résultats.

Competing interests: Les auteurs n'ont pas d'intérêts concurrents personnels ou financiers à déclarer.

Cet article a été révisé par un comité de lecture.

\section{Références}

1. Vaglio A, Salvarani C, Buzio C. Retroperitoneal fibrosis. Lancet 2006;367:241-51. https://doi.org/10.1016/S0140-6736(06)68035-5

2. Albarran J. Retention rénale par periurétérité libération externe de l'uretère. Assoc Fr Urol 1905;9:511-7.

3. Ormond JK. Bilateral ureteral obstruction due to envelopment and decompression by an inflammatory retroperitoneal process. J Urol 1948;59:1072.

4. Ha YJ, Jung SJ, Lee KH, et al. Retroperitoneal fibrosis in 27 Korean patients: Single-centre experience. J Korean Med Sci 2011;26: 985-90. https://doi.org/10.3346/ikms.2011.26.8.985

5. Zielonko J, Obolonczyk L. Retroperitoneal fibrosis with pancreatic involvement-radiological appearance. Pol J Radiol 2011;76:69-72.

6. Vaglio A, Palmisano A, Corradi D, et al. Retroperitoneal fibrosis: evolving concepts. Rheum Dis Clin North Am 2007;33:803-17. https://doi.org/10.1016/j.rdc.2007.07.013

7. Mehta A, Blodgett TM. Retroperitoneal fibrosis as a cause of positive FDG PET/CT. J Radiol Case Rep 2011;5:35-41. https://doi.org/10.3941/ircr.v5i7.722

8. Parums DV, Brown DL, Mitchinson MJ. Serum antibodies to oxidized, low-density lipoprotein and ceroid in chronic periaortitis. Arch Pathol Lab Med 1990;114:383.

9. Vaglio $\mathrm{A}$, Corradi D, Manenti L, et al. Evidence of autoimmunity in chronic pericortitis: A prospective study. Am J Med 2003;1 14:454-62. https://doi.org/10.1016/S0002-9343(03)00056-1

10. Salvarani C, Calamia KT, Matteson EL, et al. Vasculitis of the gastrointestinal tract in chronic periaortitis. Medicine (Baltimore) 2011;90:28-39. https://doi.org/10.1097/MD.0b013e318207231e

11. Vaglio A, Pipitone N, Salvarani C. Chronic periaortitis: A large-vessel vasculitis? Curr Opin Rheumatol 2011;23:1-6. https://doi.org/10.1097/BOR.0b013e328341137d

12. Umehara H, Okazaki K, Masaki Y, et al. A novel clinical entity, IgG4-related disease (IgG4RD): General concept and details. Mod Rheumatol 2012;22:1-14. https://doi.org/10.3109/s10165-01 1-0508-6

13. Zen $Y$, Onodera M, Inoue D, et al. Retroperitoneal fibrosis: A clinicopathologic study with respect to immunoglobulin G4. Am J Surg Pathol 2009;33:1833-9. https://doi.org/10.1097/PAS.0b013e3181b72882

14. Koo BS, Koh YW, Hong S, et al. Clinicopathologic characteristics of lgG4-related retroperitoneal fibrosis among patients initially diagnosed as having idiopathic retroperitoneal fibrosis. Mod Rheumatol 2015;25:194-8. https://doi.org/10.3109/14397595.2014.931908

15. Kasashima $S$, Zen Y. IgG4-related inflammatory abdominal aortic aneurysm. Curr Opin Rheumatol 2011;23:18-23. https://doi.org/10.1097/BOR.0b013e32833ee95f

16. Martorana D, Vaglio A, Greco $\mathrm{P}$, et al. Chronic periaortitis and HLA-DRB1*03: Another clue to an autoimmune origin. Arthritis Rheum 2006;55:126-30. https://doi.org/10.1002/art.21698

17. Boiardi L, Vaglio A, Nicoli D, et al. CC chemokine receptor 5 polymorphism in chronic periaortitis. Rheumatology (Oxford) 2011;50:1025-32. https://doi.org/10.1093/rheumatology/keq416

18. Goldoni $M$, Bonini $S$, Urban $M L$, et al. Asbestos and smoking as risk factors for idiopathic retroperitoneal fibrosis: A case-control study. Ann Intern Med 2014;161:181-8. https://doi.org/10.7326/M13-2648

19. Estrade V, Traxer 0 , Sibony $M$, et al. Fibrose rétropéritonéale. Encycl Méd Chir, Néphrologie-Urologie 2004;18-090-A-10, 2004. http://www.em-consulte.com/article/24120/fibrose-retroperitoneale.

20. Liu $H$, Zhang $G$, Niu $Y$, et al. Retroperitonealfibrosis: A clinical and outcome analysis of 58 cases andreview of literature. Rheumatol Int 2014;34:1665-70. https://doi.org/10.1007/s00296-014-3002-6
21. Kermani TA, Crowson CS, Achenbach SJ, et al. Idiopathic retroperitoneal fibrosis: A retrospective review of clinical presentation, treatment, and outcomes. Mayo Clin Proceedings 2011;86:297-303. https://doi.org/10.4065/mcp.2010.0663

22. Van Bommel EF, Jansen I, Hendriksz TR, et al. Idiopathic retroperitoneal fibrosis: Prospective evaluation of incidence and clinicoradiologic presentation. Medicine (Baltimore) 2009;88:193-201. https://doi.org/10.1097/MD.0b013e3181afc420

23. Ryu JH, Sekiguchi H, Yi ES. Pulmonary manifestations of immunoglobulin G4-related sclerosing disease. Eur Resp J 2012;39:180-6. https://doi.org/10.1183/09031936.0002521 1

24. Vaglio A, Strehl JD, Manger B, et al. IgG4 immune response in Churg-Strauss syndrome. Annals Rheum Dis 2012;71:390-3. https://doi.org/10.1136/ard.2011.155382

25. Barrett RL, Horrow MM, Gubernick JA, et al. US case of the day: Retroperitoneal fibrosis with perirenal involvement. RadioGraphics 1995;15:1024-6. htrps://doi.org/10.1148/radiographics.15.4.7569123

26. Cronin $C G$, Lohan DG, Blake MA, et al. Retroperitoneal fibrosis: A review of clinical features and imaging findings. Am J Roentgenol 2008;191:423-31. https://doi.org/10.2214/AJR.07.3629

27. Brun B, Laursen K, Sørensen IN, et al. CT in retroperitoneal fibrosis. Am J Roentgenol 1981;137:535-8. https://doi.org/10.2214/air.137.3.535

28. Vaglio A. Retroperitoneal fibrosis: New insights into clinical presentation and diagnosis. Medicine (Baltimore) 2009;88:208-10. https://doi.org/10.1097/MD.0b013e3181afc4dl

29. Vivas I, Nicolás Al, Velázquez P, et al. Retroperitoneal fibrosis: Typical and atypical manifestations. Br J Radiol 2000;73:214-22. https://doi.org/10.1259/bir.73.866.10884739

30. Yamada $\mathrm{H}$, Komatsu $\mathrm{R}$, Nagae $\mathrm{H}$, et al. Idiopathic retroperitoneal fibrosis with duodenal obstruction successfully treated with corticosteroids. Intern Med 1998;37:592-8. https://doi.org/10.2169/ internalmedicine.37.592

31. Surabhi VR, Menias C, Prasad SR, et al. Neoplastic and non-neoplastic proliferative disorders of the perirenal space: Cross-sectional imaging findings. RadioGraphics 2008;28:1005-17. https://doi.org/10.1148/rg.284075157

32. Szarf G, Bluemke DA. Case 83: Multifocal fibrosclerosis with mediastinal retroperitoneal involvement. Radiology 2005;235:829-32. https://doi.org/10.1148/radiol.2352030088

33. Salvarani C, Pipitone N, Versari A, et al. Positron emission tomography (PET): Evaluation of chronic periaortitis. Arthritis Rheum 2005;53:298-303. https://doi.org/10.1002/art.21074

34. Vaglio A, Greco P, Versari A, et al. Post-treatment residual tissue in idiopathic retroperitoneal fibrosis: Active residual disease or silent "scar"? A study using 18F-fluorodeoxyglucose positron emission tomography. Clin Exp Rheumatol 2005;23:231-4.

35. Piccoli GB, Consiglio V, Arena V, et al. Positron emission tomography as a tool for the 'tailored' management of retroperitoneal fibrosis: A nephrourological experience. Nephrol Dial Transplant 2010;25:2603-10. https://doi.org/10.1093/ndt/gfq051

36. Tanabe T, Tsushima K, Yasuo M, et al. IgG4- associated multifocal systemic fibrosis complicating sclerosing sialadenitis, hypophysitis, and retroperitoneal fibrosis, but lacking pancreatic involvement. Intern Med 2006;45:1243-7. https://doi.org/10.2169/internalmedicine.45.1759

37. Washino $S$, Hirai M, Matsuzaki A, et al. (18)F-fluorodeoxyglucose positron emission tomography for diagnosis and monitoring of idiopathic retroperitoneal fibrosis associated with mediastinal fibrosis. Ann Nudl Med 2010;24:225-9. htrps://doi.org/10.1007/s12149-010-0341-6

38. Young PM, Peterson JJ, Calamia KT. Hypermetabolic activity in patients with active retroperitoneal fibrosis on F-18 FDG PET: Report of three cases. Ann Nucl Med 2008;22:87-92. https://doi.org/10.1007/ s12149-007-0077-0

39. Van Bommel EF. Retroperitoneal fibrosis. Neth J Med 2002;60:231-42.

40. Khezri A, Berman HL, Rosenstein ED, et al. Spontaneous resolution of apparent radiation associated retroperitoneal fibrosis. J Clin Rheumato/ 201 1;17:436-8. https://doi.org/10.1097/RHU.0b013e31823a4e54

41. Kardar AH, Kattan $S$, Lindstedt $E$, et al. Steroid therapy for idiopathic retroperitoneal fibrosis: dose and duration. J Urol 2002;168:550-5. https://doi.org/10.1097/00005392-200208000-00031

42. Vaglio A, Palmisano A, Alberici $F$, et al. Prednisone vs. tamoxifen in patients with idiopathic retroperitoneal fibrosis: An open-label, randomized, controlled trial. Lancet 2011;378:338-46. https://doi.org/10.1016/S0140-6736(11)60934-3

43. Binder $M$, Uhl $M$, Wiech $T$, et al. Cyclophosphamide is a highly effective and safe induction therapy in chronic pericortitis: A long-term followup of 35 patients with chronic periaortitis. Ann Rheum Dis 2012;71:311-2. https://doi.org/10.1136/annrheumdis-2011-200148

44. Scheel PJ Jr, Piccini J, Rahman MH, et al. Combined prednisone and mycophenolate mofetil treatment for retroperitoneal fibrosis. J Urol 2007;178:140-3; discussion 143-4. https://doi.org/10.1016/i. juro.2007.03.057

45. Scheel PJ Jr, Feeley N, Sozio SM. Combined prednisone and mycophenolate mofetil treatment for retroperitoneal fibrosis: A case series. Ann Intern Med 2011;154:31-6. https://doi.org/10.7326/00034819-154-1-201101040-00005

46. Moroni G, Gallelli B, Banfi G, et al. Long-term outcome of idiopathic retroperitoneal fibrosis treated with surgical and/or medical approaches. Nephrol Dial Transplant 2006;21:2485-90. https://doi.org/10.1093/ndt/gfl228 
Fibrose rétropéritonéale

47. Maritati F, Corradi D, Versari A, et al. Rituximab therapy for chronic periaortitis. Ann Rheum Dis 2012;71:1262-4. https://doi.org/10.1136/annrheumdis-2011-201166

48. Heidenreich $A$, Derakhshani $P$, Neubauer $S$, et al. [Treatment outcomes in primary and secondary retroperitoneal fibrosis]. Urologe A $2000 ; 39: 141-8$. hitps://doi.org/10.1007/s001200050022

49. Seixas-Mikelus SA, Marshall SJ, Stephens DD, et al. Robot-assisted laparoscopic ureterolysis: Case report and literature review of the miniomally invasive surgical approach. JSLS 2010;14:313-9. https://doi.org/10.4293/108680810X12785289145088
50. Srinivasan AK, Richstone L, Permpongkosol S, et al. Comparison of laparoscopic with open approach for ureterolysis in patients with retroperitoneal fibrosis. J Urol 2008;179:1875-8. https://doi.org/10.1016/i. juro.2008.01.030

Correspondence: Dr. Nabil Jakhlal, Service d'Urologie B, hôpital Ibn Sina de Rabat, Maroc; doc.nabil54@gmail.com 